The Jackson Laboratory

The Mouseion at the JAXlibrary

Faculty Research 2021

Faculty Research

8-29-2021

Cardiac cellularity is dependent upon biological sex and is regulated by gonadal hormones.

Galen T Squiers

Michael A McLellan

Alexei Ilinykh

Jane Branca

Nadia Rosenthal

See next page for additional authors

Follow this and additional works at: https://mouseion.jax.org/stfb2021

Part of the Life Sciences Commons, and the Medicine and Health Sciences Commons 


\section{Authors}

Galen T Squiers, Michael A McLellan, Alexei Ilinykh, Jane Branca, Nadia Rosenthal, and Alexander R Pinto 


\title{
Cardiac cellularity is dependent upon biological sex and is regulated by gonadal hormones
}

\author{
Galen T. Squiers $\mathbb{D}^{1 \dagger}$, Micheal A. McLellan $\mathbb{D}^{2,3 \dagger}{ }^{2}$, Alexei Ilinykh $\mathbb{D}^{4}$, Jane Branca ${ }^{2}$, \\ Nadia A. Rosenthal ${ }^{2,5}$, and Alexander R. Pinto ${ }^{6,7 *}$
}

${ }^{1}$ Department of Molecular, Cellular and Developmental Biology, University of Colorado Boulder, Boulder, CO 80309, USA; ${ }^{2}$ The Jackson Laboratory, 600 Main st, Bar Harbor, ME

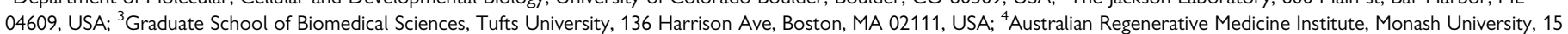

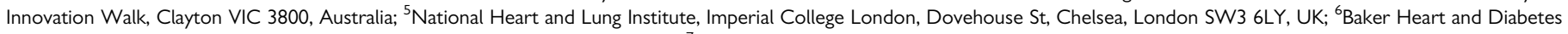

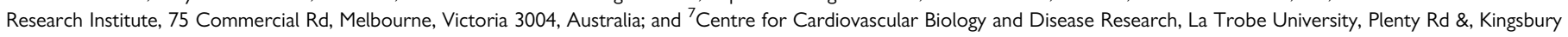
Dr, Bundoora, Victoria 3086, Australia

Received 18 May 2020; revised 10 August 2020; editorial decision 1 September 2020; accepted 5 September 2020; online publish-ahead-of-print 17 September 2020

Time for primary review: 28 days

Aims

Sex differences have been consistently identified in cardiac physiology and incidence of cardiac disease. However, the underlying biological causes for the differences remain unclear. We sought to characterize the cardiac nonmyocyte cellular landscape in female and male hearts to determine whether cellular proportion of the heart is sex-dependent and whether endocrine factors modulate the cardiac cell proportions.

Methods and results

Utilizing high-dimensional flow cytometry and immunofluorescence imaging, we found significant sex-specific differences in cellular composition of the heart in adult and juvenile mice, that develops postnatally. Removal of systemic gonadal hormones by gonadectomy results in rapid sex-specific changes in cardiac non-myocyte cellular proportions including alteration in resident mesenchymal cell and leucocyte populations, indicating gonadal hormones and their downstream targets regulate cardiac cellular composition. The ectopic reintroduction of oestrogen and testosterone to female and male mice, respectively, reverses many of these gonadectomy-induced compositional changes.

Conclusion

This work shows that the constituent cell types of the mouse heart are hormone-dependent and that the cardiac cellular landscapes are distinct in females and males, remain plastic, and can be rapidly modulated by endocrine factors. These observations have implications for strategies aiming to therapeutically alter cardiac cellular heterogeneity and underscore the importance of considering biological sex for studies examining cardiac physiology and stress responses.

\footnotetext{
†The first two authors contributed equally to the study.

*Corresponding author. Tel: +6138532 1111, E-mail: alex.pinto@baker.edu.au

Published on behalf of the European Society of Cardiology. All rights reserved. (c) The Author(s) 2020. For permissions, please email: journals.permissions@oup.com.
} 


\section{Graphical Abstract}

\section{Sex differences in cardiac cellular landscape}

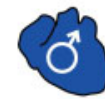

Male profile

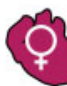

Female profile
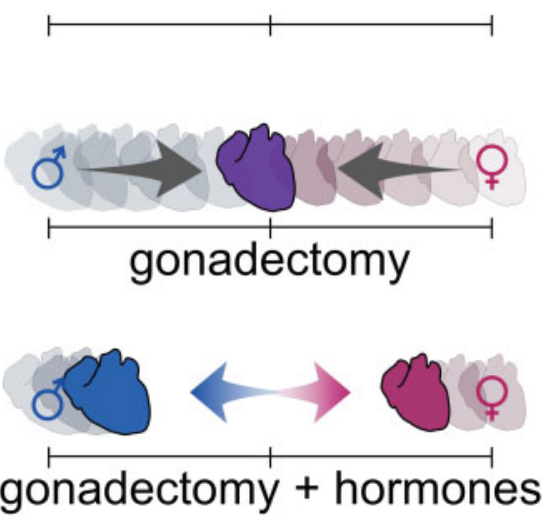

\section{Introduction}

Biological sex contributes to differences in mammalian cardiac physiology and has been identified in both the absence and the presence of tissue stress. In epidemiological and clinical data, sex differences in the prevalence and pathogenesis of cardiovascular disease (CVD) have been observed, with premenopausal women ( $<50$ years old) generally experiencing lower rates of CVD and cardiac pathology compared with age-matched males. ${ }^{1}$ Following menopause and with increasing age, the incidence of CVD in women more closely resembles that of agematched men, ${ }^{1}$ suggesting that sex hormones such as oestrogens may play an important role in protecting the heart from disease. In uninjured rodent hearts, sex-associated differences have been documented in cardiac contractility, electrophysiology, ${ }^{2}$ and gene expression. ${ }^{3-5}$ While this dichotomy is well documented, the underlying cellular basis for biological sex to drive differences in the heart is not well understood. A survey of the cardiac cellular landscape in the uninjured hearts of females and males is therefore essential to investigate how biological sex influences cardiac cell composition.

The adult mammalian heart comprises a heterogeneous mix of cells including cardiomyocytes, endothelial cells, fibroblasts, and leucocytes. Recently, we described the cardiac cellular composition of the adult mammalian heart, utilizing refined flow cytometry protocols and novel genetic tools and markers of cardiac cell types. ${ }^{6}$ Subsequently, single-cell transcriptomic analysis of the heart revealed the diversity of cardiac non- myocytes, supporting development of new approaches to survey disparate cardiac cell types. ${ }^{5}$ Here, we aimed to quantify the differences in the relative abundance of non-myocyte populations in female and male mouse hearts and the endocrine forces that regulate cardiac cellular composition. Substantial sex-dependent differences in the abundance of cardiac non-myocyte populations included resident mesenchymal cells (RMCs), T-lymphocytes, granulocytes, and subsets of cardiac tissue macrophages. The cardiac cellular ecosystem is regulated by endocrine factors, as loss of gonadal hormones such as oestrogen and testosterone dysregulated homeostatic control of cardiac cellular composition. Our finding that female and male hearts are fundamentally different at the cellular level raises important questions about the cellular basis for sex differences observed in cardiac physiology and pathology and lays a foundation for future research examining sex differences.

\section{Methods}

\subsection{Animals}

All mice (Mus musculus) were maintained in C57BL/6J background in a specific pathogen-free facility and fed standard mouse diet (Purina LabDiet 5K52) ad libitum at the Jackson Laboratory (Bar Harbor, ME, USA). All procedures had local approval (by the Jackson Laboratory Institutional Animal Care and Use Committee-IACUC) and conformed to the NIH Guide for the Care and Use of Laboratory Animals. 
Mouse tissue for all experiments was isolated after euthanizing mice by $\mathrm{CO}_{2}$ asphyxiation.

\subsection{Animal surgery and hormone treatment}

Mouse bilateral ovariectomy (OVX) and castration (Castr.) procedures were conducted by the surgical services core facility at The Jackson Laboratory, following IACUC approved protocols. Briefly, 7- to 8-weekold female or male C57BL/6J mice were anesthetized with $5 \%$ isoflurane, and maintained under 1-3\% anaesthetic administered via nosecone. Following surgery, animal welfare checks were performed daily for $72 \mathrm{~h}$ by surgical staff, and periodic checks were conducted by laboratory staff until experimental end points. For testosterone and oestrogen treatment experiments, OVX and Castr. procedures occurred simultaneous to the subcutaneous implantation of a mini-osmotic pump (Alzet Osmotic Pumps, model 2002) to supply exogenous hormones $(200 \mu \mathrm{L}$ total volume at $0.5 \mu \mathrm{L} / \mathrm{h}$ ) for 14 days. OVX mice were implanted with a pump releasing $17 \beta$-oestradiol (E2; Sigma-Aldrich) at $0.2 \mathrm{mg} / \mathrm{kg}$ of body weight/day. Castrated mice were implanted with a pump releasing testosterone propionate (TP; Sigma-Aldrich) at $1 \mathrm{mg} / \mathrm{kg}$ of body weight/day. In all experiments involving surgical intervention, mouse hearts were isolated and analysed 2 or 4 weeks after final intervention (gonadectomy and/or pump implantation; indicated by 'analysis' in Figures 3A and 4A).

\subsection{Cardiac single-cell preparation and cell staining for flow cytometry}

Preparation of cardiac single-cell suspensions and cell staining were conducted based on previously described protocols. ${ }^{6}$ Briefly, mouse hearts were isolated for single-cell preparation with atria and valves removed. Isolated hearts were finely minced and placed into a $15 \mathrm{~mL}$ Falcon tube containing $3 \mathrm{~mL}$ of digestion buffer $[2 \mathrm{mg} / \mathrm{mL}$ collagenase type IV (Worthington Biochemical Corporation) and 1.2 U/mL dispase II (SigmaAldrich) in Dulbecco's phosphate-buffered saline (DPBS) supplemented with $0.9 \mathrm{mmol} / \mathrm{L} \mathrm{CaCl}_{2}$ ]. Tissue was incubated at $37^{\circ} \mathrm{C}$ for $45 \mathrm{~min}$ with tissue triturated every $15 \mathrm{~min}$. All cell suspensions were filtered using a $70 \mu \mathrm{m}$ cell strainer. Filtered suspensions were placed in a $15 \mathrm{~mL}$ tube with $\sim 10 \mathrm{~mL}$ DPBS and centrifuged at $200 \mathrm{~g}$ for $20 \mathrm{~min}$ with centrifuge brakes deactivated to remove small tissue debris. Cell pellets were resuspended in $200-250 \mu \mathrm{L}$ of $2 \% \mathrm{FBS} / \mathrm{HBSS}$ solution before staining with various antibodies or dyes for flow cytometry.

Antibody staining (Supplementary material online, Table S1) was conducted in $100 \mu \mathrm{L}$ of single-cell suspension (in $2 \% \mathrm{FBS} / \mathrm{HBSS}$ ) after FC receptor blocking with CD16/CD32 antibody. Following $1 \mathrm{~h}$ antibody incubation at $4^{\circ} \mathrm{C}$, calcein (calcein-AM; Thermo-Fischer Scientific) was added to antibody/cell suspension at a final concentration of $5 \mu \mathrm{M}$. Samples with calcein were incubated for $10 \mathrm{~min}$ in a $37^{\circ} \mathrm{C}$ water bath, before chilling samples on ice. Samples were washed and re-suspended in $2 \% \mathrm{FBS} / \mathrm{HBSS}$ with or without the viability dyes DAPI or 7-AAD. All flow cytometry was conducted on LSR II Fortessa Flow Cytometer (BD Biosciences) or FACS Symphony A5 Flow Cytometer (BD Biosciences). For compensation of fluorescence spectral overlap, UltraComp eBeads (eBioscience, Inc.) were used following the manufacturer's protocol. FCS 3.0 files generated by flow cytometry were initially processed using Flowjo software (Flowjo, LLC) for automated compensation. To gain better insight towards sex-related differences in cardiac cell types, we performed unsupervised cell population clustering using the spanningtree progression of density-normalized events (SPADE) algorithm on populations indicated in Supplementary material online, Figures S1 and S3, clustering cells based on markers indicated. ${ }^{6,7}$ All SPADE analyses were conducted using the Cytobank Premium platform with the target number of nodes set at 200 and down-sampled events target set at $100 \%$. Dendrogram nodes (circles) represent phenotypically identical/similar cell populations and connectedness indicates phenotypic relation of nodes to each other. Subjective (non-SPADE) flow cytometry analyses were also conducted where indicated. Dendrograms generated using SPADE enable visualization of the entire cardiac non-myocyte cellular landscape. ${ }^{6}$ Moreover, relative frequencies of phenotypically similar populations could be identified using this approach. Nodes of SPADE dendrograms represent phenotypically identical/similar cell populations and connectedness indicates phenotypic relation of nodes to each other.

\subsection{Immunofluorescence staining of cardiac tissue sections}

Formaldehyde-fixed hearts were isolated from female and male mice and embedded in paraffin. About $5 \mu \mathrm{m}$ thick mid-ventricular sections were prepared and stained on glass slides. Briefly, samples were rehydrated and boiled in a microwave for $15 \mathrm{~min}$ in Tris-EDTA for antigen retrieval and allowed to cool to room temperature for preparation for antibody staining. Sections were washed with $0.05 \%$ Tween 20 and then permeabilized with $0.5 \%$ Triton $X-100$. Non-specific staining was blocked ( $2 \%$ goat serum, $1 \%$ BSA, $0.1 \%$ cold fish skin gelatin, $0.1 \%$ Triton $\mathrm{X}-100,0.05 \%$ Tween-20, 0.05\% sodium azide, 0.01 M PBS, $\mathrm{pH} 7.2$ ) for $1 \mathrm{~h}$ at room temperature. Primary antibodies (Supplementary material online, Table S1) were diluted in blocking solution and stained samples overnight at $4^{\circ} \mathrm{C}$. Samples were then incubated with secondary antibodies for $2 \mathrm{~h}$ at room temperature. Finally, samples were treated with DAPI and mounted before images were acquired using Leica SP8 confocal laser scanning microscope and processed using FIJI image analysis software (version: 2.0.0-rc-69/1.52n).

\subsection{Single-cell RNA-sequencing data analyses}

Analysis of a previously published cardiac single-cell RNA-seq dataset ${ }^{5}$ and heat mapping of gene expression (as in Supplementary material online, Figure S2) was performed using the Seurat v3 R package.,

\subsection{Statistical analyses}

All statistical analyses were conducted using either Student's $t$-test or ANOVA (two-way) using ggpubr package on $\mathrm{R}$ to compare differences between females and males or other experimental cohorts or Prism software (GraphPad Software, Inc). Differences where $P \leq 0.05$ were considered statistically significant.

\section{Results}

To determine sex differences in cellular abundances of the mammalian heart, and detect endocrine factors regulating cardiac cellular composition, we examined ventricular tissue from adult female and male mice. We performed flow cytometric analyses on isolated metabolically active non-myocytes (Supplementary material online, Figure S1A) ${ }^{6}$ to determine the relative frequencies of broad cell classes including endothelial cells (ECs: CD $\left.31^{+} \mathrm{CD} 45^{-}\right)$, RMCs (CD31-CD45'), and leucocytes (Leucs: $\mathrm{CD} 31^{-} \mathrm{CD} 45^{+}$; Figure $1 \mathrm{~A}$ and $\mathrm{B}$ ). The relative cell proportions of both the EC and the RMC compartments differed significantly between the sexes, while the proportion of total leucocytes was not different. To determine whether this was the result of a reduced number of ECs or an 
A

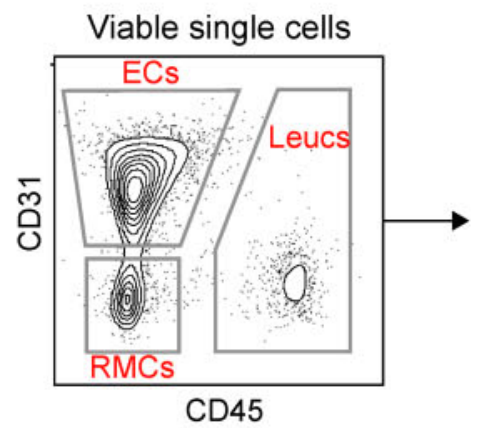

B

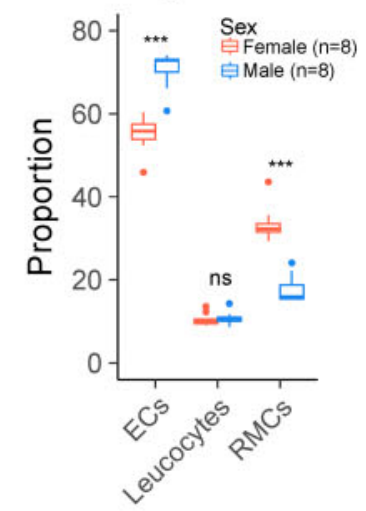

Count

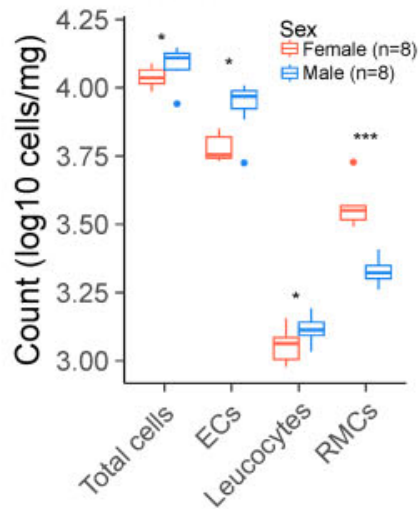

C

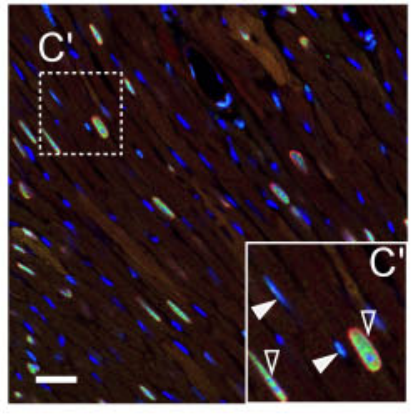

E

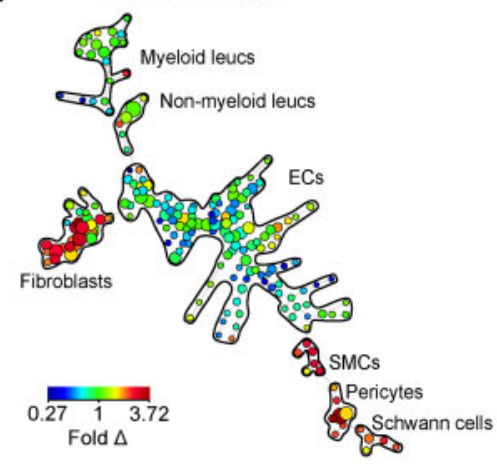

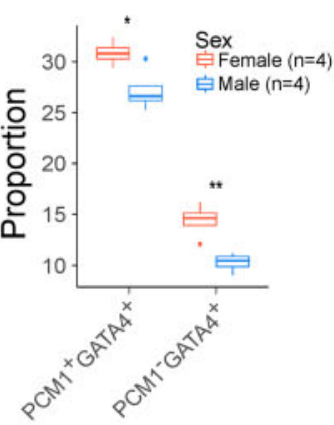

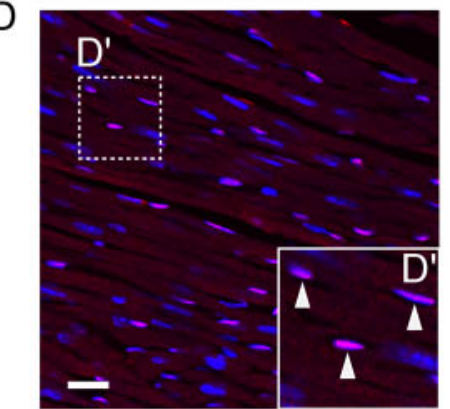

F

RMCs -proportion

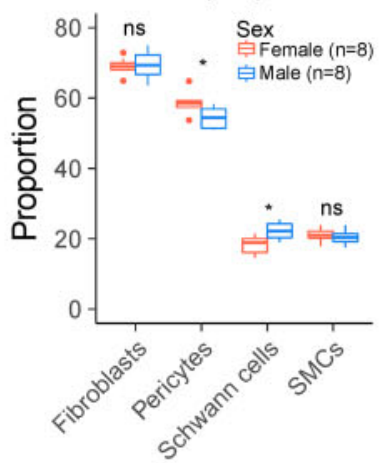

RMCs - count

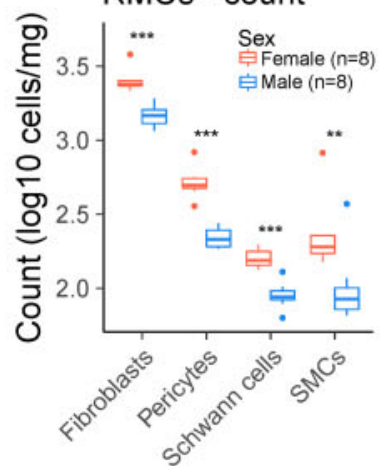

$\mathrm{G} \quad$ Leucocyte - proportion

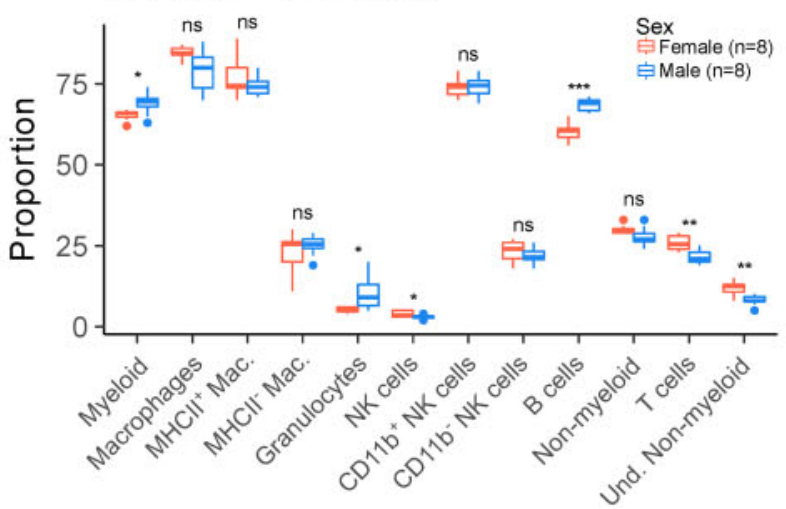

Leucocyte - count

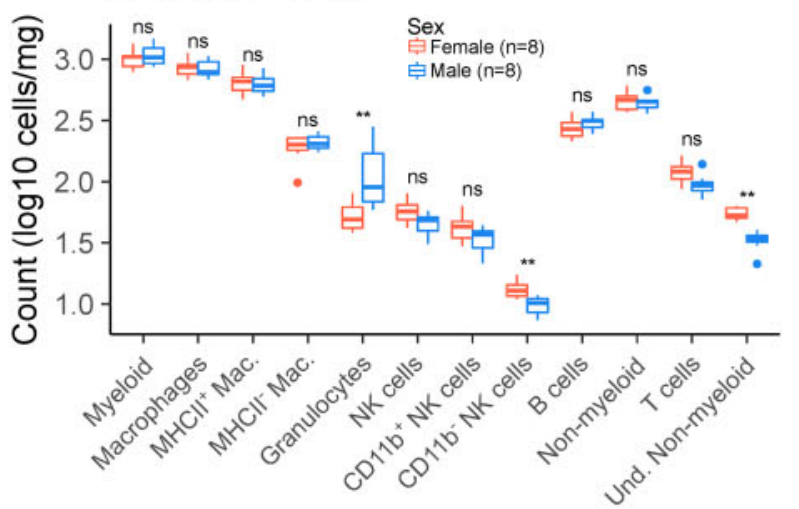

Figure I Cellular composition of the female and male heart is distinct. (A) Flow cytometry workflow for analysis of broad non-myocyte cardiac cell populations (left) and quantification of $\mathrm{CD} 31^{+} \mathrm{CD} 45^{-}$(ECs), $C D 45^{+}$(leucocytes), and CD31 CD45 (RMCs) cells as a proportion of non-myocytes. (B) Proportion (left panel) and absolute numbers (right panel) of cells corresponding to ECs, leucocytes, and RMCs. (C) Immunohistochemical analysis of proportion of nuclei corresponding to cardiomyocytes $\left(\mathrm{PCM}^{+} \mathrm{GATA}^{+}\right.$) or RMCs (PCM-GATA4 ${ }^{+}$). Scale bar indicates $25 \mu \mathrm{m}$. (D) Immunohistochemical 
increased number of RMCs, we normalized cell counts for each population (determined by flow cytometry) to input tissue mass used for single-cell preparation (Figure 1B). We found absolute numbers of ECs in both females and males were similar, whereas the absolute number of RMCs was significantly higher ( $\sim 1.6$-fold) in females compared to males.

To corroborate our flow cytometry results, we undertook immunohistochemical analysis of cardiac ventricles from female and male mice. To identify gene expression markers for RMCs that are suitable for immunohistochemical detection, we analysed a previously published single-cell transcriptomic dataset of cardiac non-myocytes. ${ }^{5}$ We found Gata4 expression is relatively restricted to RMCs (fibroblasts, smooth muscle cells, and pericytes), although expression was not enriched in Schwann cells (Supplementary material online, Figure S2). Gata4 is also enriched in cardiomyocytes, therefore, in addition to Gata4, we stained samples for the cardiomyocyte marker PCM1. ${ }^{10}$ Moreover, our analysis of Dach1 expression confirmed that it is a suitable marker for detecting endothelial cells (Supplementary material online, Figure S2). ${ }^{6}$ Enumeration of nuclei showed a disparity between female and male hearts in proportion of total nuclei corresponding to cardiomyocytes $\left(\mathrm{PCM}^{+}{ }^{+} \mathrm{GATA}^{+}\right)$and RMCs (PCM GATA ${ }^{+}$; Figure $1 \mathrm{C}$ and D). Similarly, by staining cardiac sections for DACH1, a nuclear marker of endothelial cells (Supplementary material online, Figure S2), ${ }^{6}$ we found that male hearts comprised a higher proportion of nuclei corresponding to endothelial cells ( $\mathrm{DACH} 1^{+}$nuclei, $\left.P=0.093\right)$. Therefore, by examining both cell proportion (by flow cytometry and histology) and absolute cell numbers, which account for differences in heart cell mass, our results show sex-specific differences in the proportions of cells found in male and female mouse hearts.

Using SPADE analysis, ${ }^{6}, 7$ different cell populations can be clustered as 'branches' on the dendrograms; providing a powerful tool to identify cardiac cell types and visualize cell abundance differences. SPADE analysis was conducted on metabolically active, viable, single cells (Supplementary material online, Figure S1B), and cell populations were clustered based on EC (CD31), RMC (MEFSK4, CD59a, CD146, CD39a) and leucocyte (CD45, CD11b) markers. Objective classification of the dendrograms was undertaken by identifying branches corresponding to ECs (CD31hiCD45-), RMCs (CD31-CD45-), and leucocytes (CD45+CD11b+; Figure 1E). Ratiometric SPADE colour-mapping of female hearts relative to cell frequencies in male hearts showed widespread differences within broad cardiac cell classes (Figure $1 E$ ), pointing to sex differences in cell abundances not identified by using markers for the major cell populations.

To determine the extent of sex differences in the cardiac RMC compartment, we examined relative proportions and absolute numbers of fibroblasts, pericytes, Schwann cells, and SMCs (Figure $1 F$ and Supplementary material online, Figure S1) by flow cytometry. ${ }^{5}$ This analysis revealed disparity in proportions of pericytes and Schwann cells, whereas analysis of absolute cell counts showed that all RMC cell numbers were higher in hearts of female mice compared to males consistent with our microscopy data (Figure 1C).

Initial definition of broad cell classes and ratiometric SPADE analyses suggested that the relative abundance of some leucocyte subpopulations is determined by biological sex. The relative abundance of wide arrays of leucocyte subpopulations (Figure $1 \mathrm{G}$ and Supplementary material online, Figure S1) showed males have increased proportions of total myeloid cells, granulocytes, and B cells, whereas females have increased proportions of NK cells, T cells, and Undetermined non-myeloid (Und. Non-myeloid) cells (Figure $1 G$ ). Further examination of absolute numbers of cells showed increases in granulocytes in males and increases in CD11b NK cells and Und. Non-myeloid cells in females (Figure $1 \mathrm{G}$ ). Together these results show that sex differences exist in multiple cellular compartments of the mouse heart.

To determine the ontogeny of the sex differences in cardiac cellular composition, we examined female and male mouse hearts at either 1 or 4 weeks of age (Figure 2). Using an antibody panel that identifies a wide array of cardiac cell subsets, including those within the RMC and leucocyte compartments, we undertook high-dimensional flow cytometric analyses (Supplementary material online, Figure S3) coupled with unsupervised cell clustering. Ratiometric (female:male) SPADE analysis revealed sex-dependent differences in cell proportions in multiple cell compartments in 4-week-old mice, particularly within the RMC compartment, whereas no difference was observed in 1week-old animals (Figure 2A). Subjective gating to identify changes in broad cell classes (ECs, RMCs, and leucocytes) showed substantial sex-dependent proportional differences in female ECs $(\sim 10 \%$ lower proportion) and RMCs ( $\sim 11 \%$ higher proportion) relative to males in 4-week-old mice (Figure 2B), mirroring relative broad cell differences observed in adult animals (Figure 1). In contrast, no significant differences in broad cell classes were observed in 1-week-old mice (Figure 2A). Moreover, we identified sex-dependent differences in the relative abundance of cardiac fibroblasts (MEFSK4 ${ }^{\text {hi }}$ RMCs) (Figure 2C), myeloid cells as a percentage of leucocytes (Figure 2D), and subsets of non-myeloid leucocytes (B cells and T cells) in 4-weekold mice (Figure 2F) but not in 1-week-old animals. Collectively, our data suggest that sex-dependent differences in cardiac cellularity principally develop in mice after 1 week of age.

While flow cytometric analyses revealed that the cellular composition of female and male hearts differ in a number of cellular compartments, the factors that govern these distinct cellular signatures are unclear. We hypothesized that endocrine cues, including sex hormones, may contribute to the development of differences based on biological sex in cardiac cellular composition and may regulate relative cell frequencies in cardiac homeostasis. Analysis of cardiac cellular composition in mice that had undergone bilateral ovariectomy $(\mathrm{OVX})$ or castration (Castr.) at

\section{Figure I Continued}

analysis of proportion of nuclei corresponding to endothelial cells (DACH1 ${ }^{+}$nuclei). Scale bar indicates $25 \mu \mathrm{m}$. (E) Ratiometric (female:male) SPADE analysis of broad cardiac cell types in 10-week-old mice. Heat map indicates fold difference (fold $\Delta$ ) of cell populations in female hearts compared to male hearts. Nodes (circles) of SPADE dendrograms represent phenotypically identical/similar cell populations, size of nodes indicates relative abundances of cells represented by the node, and connectedness indicates phenotypic relation of nodes to each other. See Supplementary material online, Figure S1, for markers used for cell clustering. $(F)$ Proportions and absolute numbers of RMCs determined by flow cytometry. ( $G$ ) Proportions and absolute numbers of cardiac leucocytes. See Supplementary material online, Figure S1, for all flow cytometry gating parameters and markers used for clustering. All data analysed using Student's $t$-test. Where exact values are not provided, significance indicated as $*(P \leq 0.05)$, $* *(P \leq 0.01)$, *** $(P \leq 0.001)$, and $* * * *(P \leq 0.0001)$. Whiskers of box and whisker plots represent the highest and the lowest values, except when a value is beyond the range of 1.5 inter-quartile. 

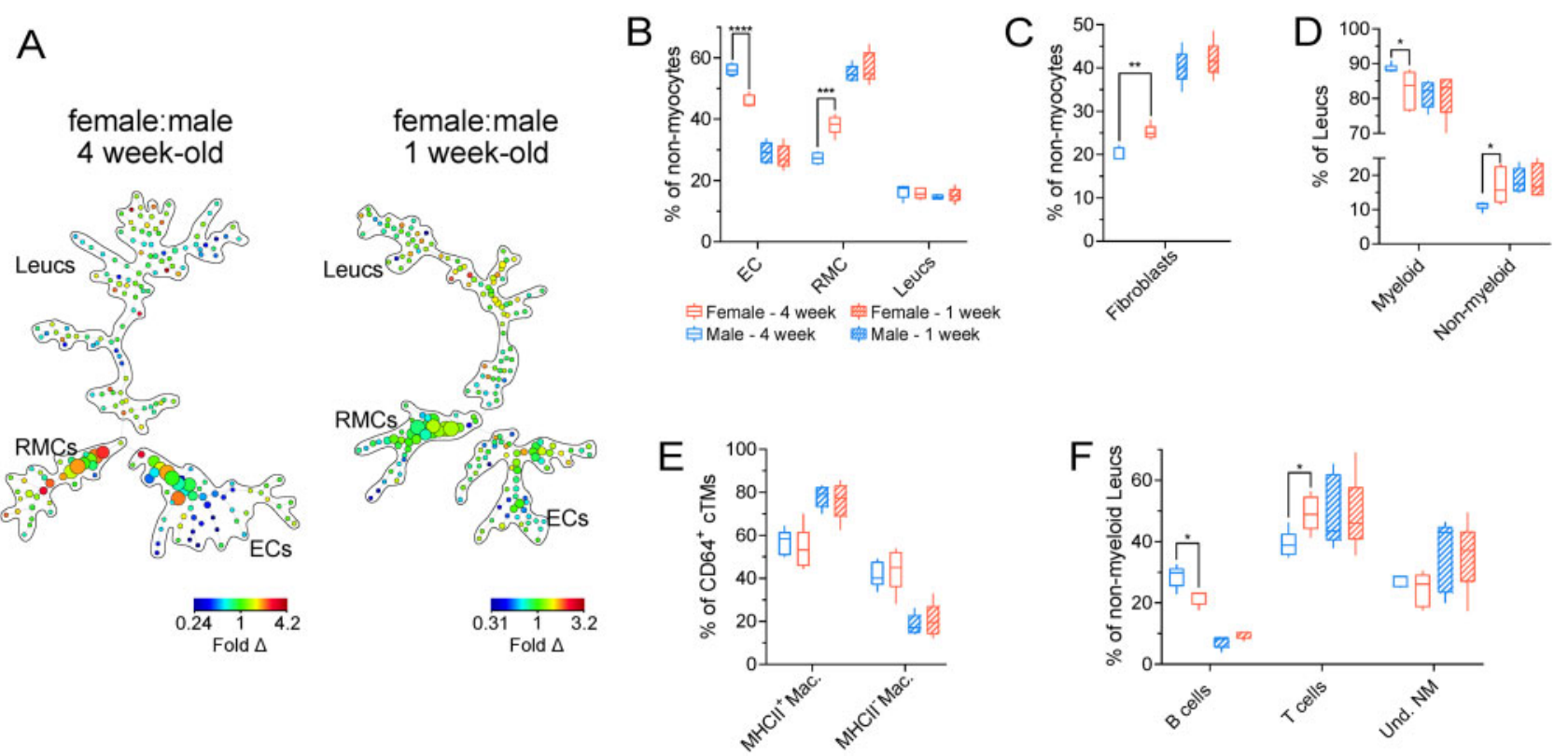

Figure 2 Differences based on biological sex in cardiac cellular composition principally develops after birth. (A) Ratiometric (female:male) SPADE analysis of broad cardiac cell types in 4-week-old (left) and 1-week-old (right) mice. Heat map indicates fold difference (fold $\Delta$ ) of cell populations in female hearts compared to male hearts. (B) Proportions of ECs, RMCs, and Leucs (leucocytes) relative to total non-myocytes in 4- and 1-week-old males and females. (C) Proportions of fibroblasts relative to total non-myocytes in 4- and 1-week-old males and females. (D) Proportions of myeloid $(C D 45+C D 11 b+)$ and non-myeloid (CD45+CD11b-) Leucs relative to total Leucs in 4- and 1-week-old males and females. (E) Proportions of MHClland $\mathrm{MHCll}+$ macrophages (Macs: CD45+CD11b+Ly6G-CD64+) relative to total macrophages in 4- and 1-week-old males and females. $(F)$

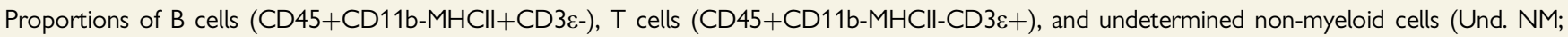
CD45+CD11b-MHCII-CD3ع-) relative to total non-myeloid Leucs in 4- and 1-week-old males and females. See Supplementary material online, Figure $S 3$, for all flow cytometry gating parameters and markers used for SPADE clustering. $n=5$ per group; all statistical analysis performed using Student's $t$-test with statistical significance indicated as $*(P \leq 0.05)$, ** $(P \leq 0.01)$, *** $(P \leq 0.001)$, and $* * * *(P \leq 0.0001)$. Whiskers of box and whisker plots represent the highest and the lowest values.

7 weeks of age relative to sham controls (Figure $3 A$ ) revealed a timedependent increase in body weight and heart weight in cohorts of OVX mice compared to controls, and corresponding decreases in cohorts of castrated mice (Figure 3B). To determine the effect of gonadectomy on the cardiac cellular landscape, we undertook flow cytometric analyses using an antibody panel to identify a wide range of cell types comprising EC, RMC, and leucocyte compartments. Ratiometric SPADE analysis of gonadectomized animals relative to controls showed that gonadectomy in both females and males reconfigured the cardiac composition of multiple cellular compartments within 2 weeks (Figure 3C). Quantitative analysis of the relative abundance of broad cell types showed that castration led to an increase in the levels of RMCs (including fibroblasts) while ovariectomy reduced the number of RMCs relative to sham controls (Figure 3D). These data suggest that female and male gonadal hormones have opposite regulatory effects on the RMC compartment. In contrast to the effect of gonadal hormones on cardiac RMCs, both castration and ovariectomy had a similar effect on many leucocyte subsets, including higher levels of non-myeloid cells, substantially lower levels of granulocytes and a decreased proportion of $\mathrm{MHC}^{-}$macrophages.

To gain a more global perspective of the effect of gonadectomy on the sex differences of the cardiac cellular landscape, we performed principal component analysis using the relative cell abundance data for each cell population plotted in Figure 3D (Figure 3E). As expected, our
PCA analysis showed that the cellular compositions of the male and female hearts are distinct and that from 2 weeks post-gonadectomy the cellular landscapes of the two populations become similar. Intriguingly, cellular profiles of female and male hearts resemble each other 4 weeks after gonadectomy, however, all cohorts - non-gonadectomized, 2 weeks gonadectomized, and 4 weeks gonadectomizeddisplay distinct cellular profiles (Figure 3E). Together, our findings indicate that gonadal hormones are essential for maintaining the normal cardiac cellular landscape. Furthermore, sex hormones regulate the cardiac cellular composition in a cell type-dependent manner with some cardiac cells regulated in opposite directions by male and female gonadal hormones (e.g. RMCs) while other cell types (e.g. leucocyte subsets) are regulated in the same way.

Next, we sought to determine whether oestrogen and testosterone signalling, in particular, are responsible for altering cardiac cellular proportions. To test this, we treated Castr. or OVX mice for 2 weeks with either E2 or TP, respectively (Figure 4A). Testosterone treatment reversed the loss of body weight and heart weight associated with castration (Figures $4 B$ and $3 B$ ). At the end of the experimental protocol (4 weeks), we conducted a ratiometric SPADE analysis of cardiac cellular composition (Figure 4C) and found that both E2 and TP treatments affect multiple cellular compartments and had opposing effects on the levels of RMCs and 
A

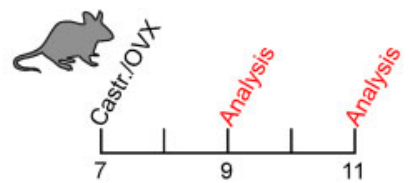

B

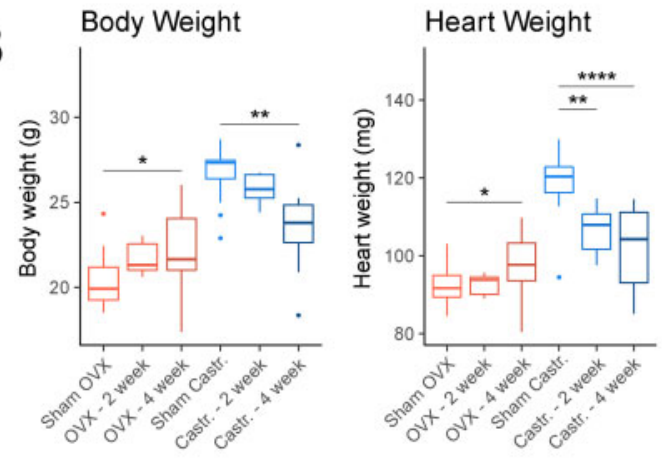

D

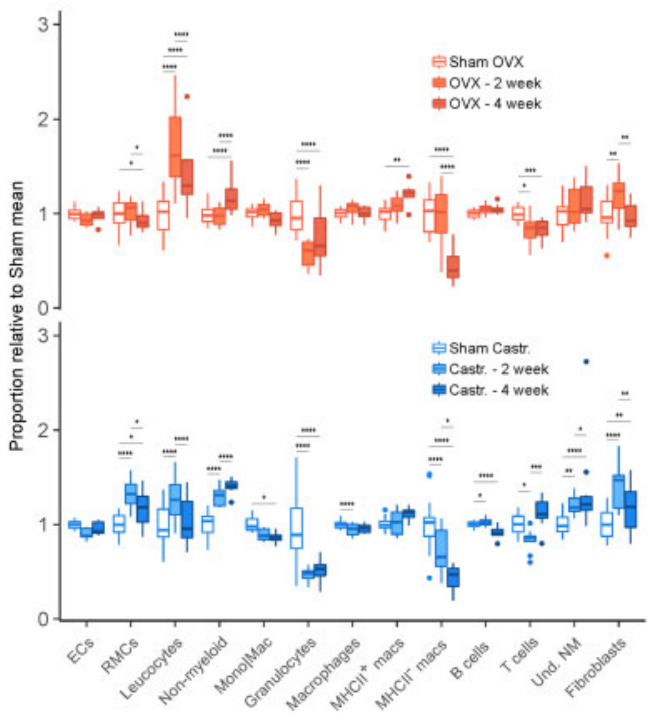

C Castr.:Sham Castr.

OVX:Sham OVX
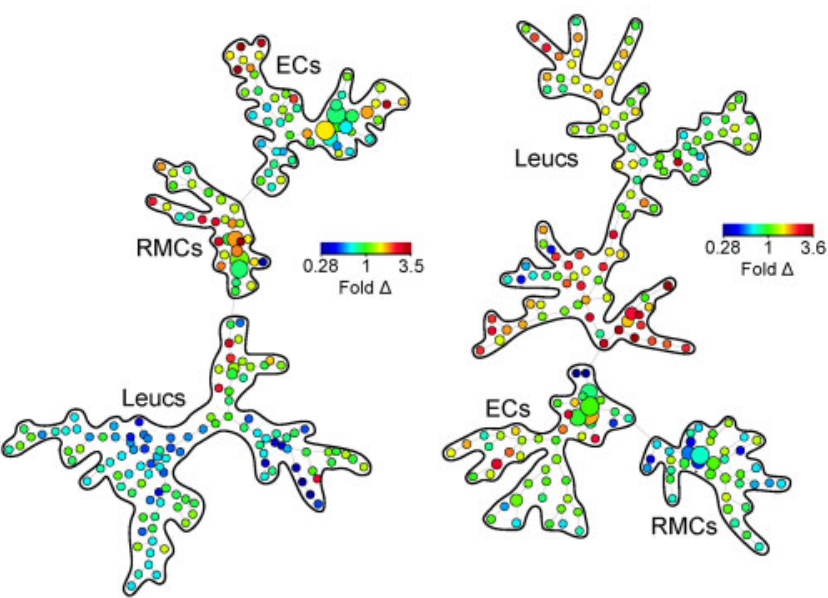

E

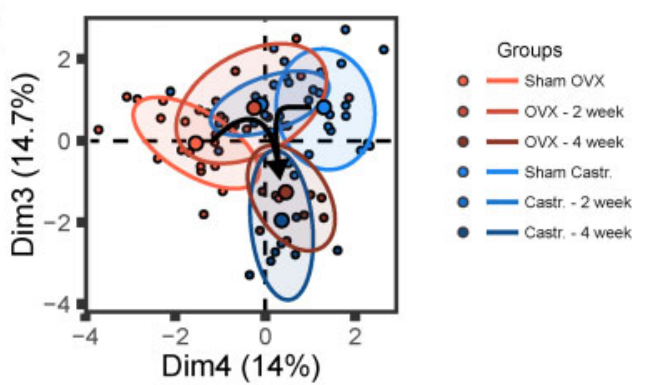

Figure 3 Gonadal hormones maintain the homeostatic cardiac cellular signature in female and male hearts. (A) Experimental set-up for mouse ovariectomy (OVX) or castration (Castr.). (B) Body weight (left) and heart weight (right) measurements following sham surgery or gonadectomy. (C) Ratiometric (gonadectomy: sham surgery) SPADE analysis of broad cardiac cell types. Heatmap indicates fold difference (fold $\Delta$ ) of cell populations in gonadectomized animals compared to sham controls. (D) Relative abundance of broad cell types (ECs, RMCs, and Leucs), broad leucocyte subsets (non-myeloid, Mono|Mac, granulocytes), macrophage subsets ( $\mathrm{MHCll}^{+}$macrophages and $\mathrm{MHCll}^{-}$macrophages), and non-myeloid subsets (B cells, $\mathrm{T}$ cells, and Und. NM cells) between gonadectomized animals and sham surgery controls. ECs (CD31 $\left.{ }^{+} \mathrm{CD} 45^{-}\right)$; RMCs $\left(\mathrm{CD} 31^{-} \mathrm{CD} 45^{-}\right)$, leucocytes (Leucs; $\left.\mathrm{CD} 45^{+}\right)$; monocytes/

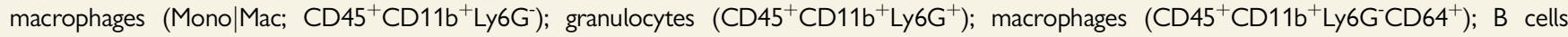
$\left(\mathrm{CD} 45^{+} \mathrm{CD} 11 \mathrm{~b}^{-} \mathrm{MHCll}^{+} \mathrm{CD} 3 \varepsilon^{-}\right)$; T cells $\left(\mathrm{CD}^{+} 5^{+} \mathrm{CD} 11 \mathrm{~b}^{-} \mathrm{MHCll}^{-} \mathrm{CD} 3 \varepsilon^{+}\right)$; undetermined non-myeloid cells (Und. NM; CD45 $\left.{ }^{+} \mathrm{CD} 11 \mathrm{~b}^{-} \mathrm{MHCll}^{-} \mathrm{CD} 3 \varepsilon^{-}\right)$. (E) Principal component analysis cellular composition of sham and gonadectomized mice. Principal components that best differentiated female and male nongonadectomized cohorts were chosen. Parenthesis on axis labels indicate variation represented by principal component. See Supplementary material online, Figure S3, for all flow cytometry gating parameters and markers used for SPADE clustering. $n=13-15$; all statistical analysis performed using Student's $t$-test with statistical significance indicated as $*(P \leq 0.05)$, ** $(P \leq 0.01), * * *(P \leq 0.001)$, and $* * * *(P \leq 0.0001)$. NS, $P$-value $>0.05$. Whiskers of box and whisker plots represent the highest and the lowest values, except when a value is beyond the range of 1.5 inter-quartile.

ECs (Figure 4D). Indeed, PCA analysis of relative abundances of cardiac cell types indicates E2 and TP infusion, respectively, partially reverses the changes in cardiac cellular composition that follows ovariectomy and castration (Figure 4E). These data suggest that gonadal hormones act on the heart to regulate cardiac cellular heterogeneity.

\section{Discussion}

While experimental and epidemiological studies point to sex differences in cardiac physiology and pathology, ${ }^{1,11,12}$ fundamental differences in the diversity and abundance of cells in female and male hearts have not been previously described. Here, we show that the cardiac cellular landscapes 
A

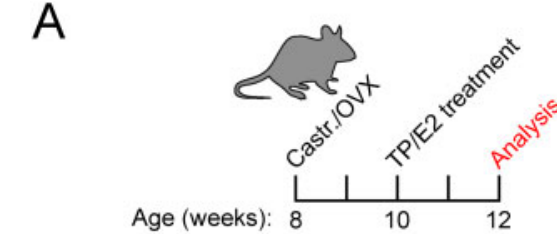

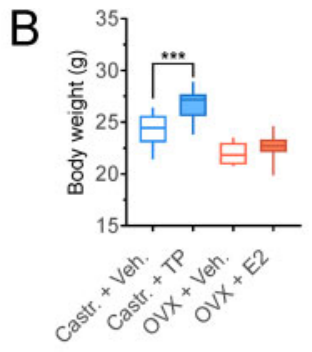

C

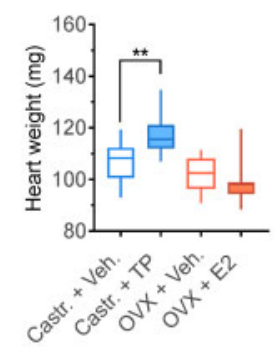

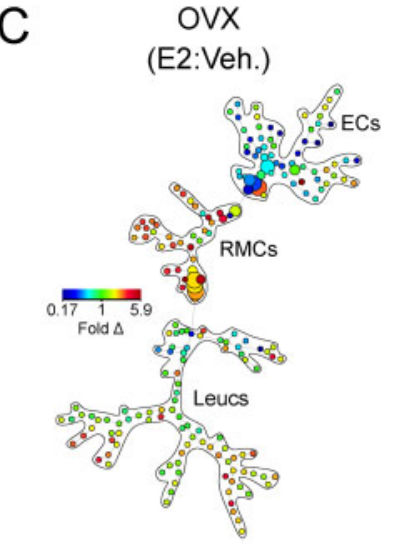

Castrated

(TP:Veh.)

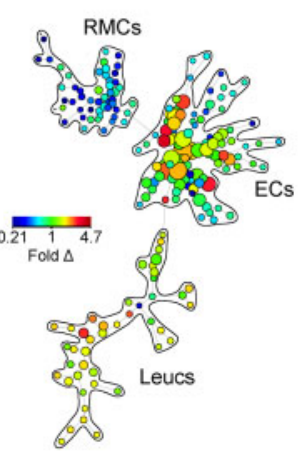

Figure 4 Gonadal oestrogen and testosterone regulate cardiac resident mesenchymal levels (RMCs) and leucocyte subsets. ( $A$ ) Experimental set-up for mouse OVX or Castr. followed by Veh. or TP/oestradiol (E2) treatment. (B) Body weight (left) and heart weight (right) measurements following gonadectomy with vehicle or TP/E2 treatment. (C) Ratiometric (TP: vehicle control and E2: vehicle control, Castr. males and OVX females, respectively) SPADE analysis of broad cardiac cell types. Heat map indicates fold difference (fold $\Delta$ ) of cell populations in gonadectomized animals with TP or E2 treatment relative to gonadectomized animals with vehicle control treatment. (D) Relative abundance of broad cell types (ECs, RMCs, and Leucs), broad leucocyte subsets (non-myeloid, Mono|Mac., granulocytes), cardiac tissue macrophage subsets (MHCII macrophages and $\mathrm{MHCll}^{-}$macrophages) and nonmyeloid subsets ( $B$ cells, T cells, and Und. NM cells) between gonadectomized animals treated with TP/E2 and gonadectomized animals treated with vehicle control. (E) Principal component analysis cellular composition of non-OVX (control), OVX, and OVX+E2 treatment cohorts. Arrows indicate movement of cellular composition signatures following ovariectomy and E2 treatment following ovariectomy. $(F)$ Principal component analysis cellular composition of non-Castr. (control), Castr., and Castr.+TP treatment cohorts. Arrows indicate movement of cellular composition signatures following castration and TP treatment following castration. See Supplementary material online, Figure S3, for all flow cytometry gating parameters and markers

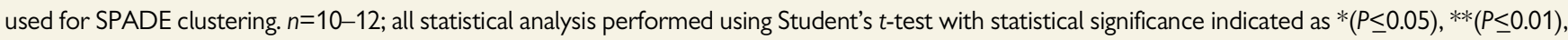
$* * *(P \leq 0.001)$, and **** $(P \leq 0.0001)$. Whiskers of box and whisker plots represent the highest and the lowest values, except when a value is beyond the range of 1.5 inter-quartile.

of female and male mice are distinct. Sex-specific differences are detectable in juvenile mice (after 1 week of age) and persist in older animals. Moreover, we find that gonadal hormones are key regulators driving the differences in cardiac cellular composition, and demonstrate the plasticity of the cardiac cellular landscape.

A number of cardiac sex-dependent characteristics have been previously described in both humans and experimental models. These include increased ventricular stiffness and HFpEF in later life in human females, ${ }^{13}$ and female hearts having dampened levels of inflammation compared to male hearts with decreased cardiomyocyte death in failing hearts. ${ }^{14}$ In uninjured rodent hearts, sex-associated differences have been documented in cardiac contractility, electrophysiology, 2,15,16 and gene expression, ${ }^{3,4,17,18}$ including in embryonic stem cells. ${ }^{19}$ Compared to males, young females have reduced mortality and display improved cardiac function after ischaemic injury, ${ }^{20,21}$ show reduced levels of fibrosis in a model of cardiac hypertrophy, ${ }^{22}$ and lower risk of rupture following MI. $^{18,23}$

This invites further research to determine whether sex-specific differences in cardiac cellularity, as described in this study, play a role in these sex differences. The differences in abundances of RMC compartment and fibroblasts, specifically, may be important here. Fibroblasts are the principal extracellular matrix producing cell population in the heart ${ }^{24,25}$ and may play a role in increased cardiac stiffness in females. Independent of ECM remodelling, fibroblasts play a multitude of functions in cardiac homeostasis and disease, ${ }^{26,27}$ including key support roles for multiple cardiac cell types. ${ }^{5}$ Cardiac fibroblasts possess salutary mesenchymal 
stem cell-like properties and release paracrine factors that may dampen deleterious inflammatory responses and promote cardiomyocyte survival. 28,29

Our study also detected differences in leucocyte subpopulations. These include greater levels of $\mathrm{T}$ cells and reduced levels of granulocytes in females consistent with observation from others relating to sex differences in circulating leucocyte levels. ${ }^{30} \mathrm{~T}$ cells may confer both beneficial and harmful effects, depending on the T-cell subset and context. ${ }^{31}$ For example, Foxp $3^{+} \mathrm{CD}^{+} \mathrm{T}$ regulatory cells improve cardiac healing after myocardial infarction by reducing infarct size ${ }^{32}$ and modulating the inflammatory cellular milieu. ${ }^{32,33}$ Conversely, $\mathrm{CD}^{+} \mathrm{T}$ cells contribute to increased infarct size after experimental myocardial injury in an IFN- $\gamma$ dependent manner. ${ }^{34}$ Further research is required to investigate which T-cell subsets are increased in the female in cardiac homeostasis and whether these $T$ cells display beneficial properties relative to males. Granulocytes, especially neutrophils, are myeloid leucocytes that are recruited to the myocardium within 1-2 days of injury and are strongly correlated with poor cardiac function. ${ }^{35,36}$ Neutrophils are thought to contribute to tissue damage by release of reactive oxygen species, proteases, and inflammatory cytokines. ${ }^{37}$ Very little is known about the role of granulocytes in cardiac homeostasis but we speculate that the presence of fewer neutrophils in female hearts is favourable for cardiac function, particularly after cardiac stress.

Beyond the documentation of extensive differences in cellular heterogeneity between female and male mouse hearts, our data suggest that gonadal hormones are key regulators of cardiac cellularity. The role of sex hormones such as oestrogens and testosterone in regulating cellular proliferation, differentiation, and behaviour has been previously documented. However, no studies to date have tested the modulation of cardiac cellular heterogeneity by endocrine cues. Here, we show that both ovariectomy and castration uniquely alter the relative abundance of cardiac non-myocytes. It is particularly noteworthy that the differences in RMC proportions-a defining feature of sex differences in cardiac cellular composition - are regulated by both female and male gonadal hormones, with castration increasing, and ovariectomy decreasing, RMC levels. Treatment of castrated and ovariectomized mice with testosterone or oestrogen (respectively) partly reverses RMC levels back to the baseline. Moreover, similar hormone-dependent patterns are also observed for other cell populations such as granulocytes, $\mathrm{MHCll}^{-}$macrophages, and $\mathrm{T}$ cells. These data demonstrate that sex hormones are critical determinants of cardiac cellular composition, operating in a cell type-dependent manner.

We also identified shifts in cell abundance and phenotype that were dependent on gonadal hormone irrespective of sex. Gonadectomy in both females and males shifts macrophage proportions to $\mathrm{MHCll}+$ phenotype, although macrophage numbers are not significantly altered. We observed the reversal of this upon infusion of E2 or TP to ovariectomized or castrated mice, respectively. This observation suggests that circulating gonadal hormones are essential for maintaining macrophage phenotypic heterogeneity in the heart. Similarly, we also noted that granulocytes decrease in proportion after gonadectomy, although this reduction was not significantly reversed upon infusion of ectopic hormone. Given that macrophages have been implicated in tissue remodelling in a number of contexts, ${ }^{38}$ it is possible that hormonal transformation of the cellular landscape of the heart is dependent on macrophage activity.

Our findings offer a foundation for further investigation into the influence of biological sex on cardiac cellular heterogeneity. Recent research has pointed to extensive sex differences in cardiac gene expression within cardiac cell populations, 5,24 however, the impact of these differences on cardiac biology and stress responses remains unclear. Moreover, the precise mechanisms by which sex hormones and perhaps other endocrine mediators alter cardiac cellular diversity and proportions remain to be examined. Various cell types such as macrophages, ${ }^{39,40}$ fibroblasts, ${ }^{41,42}$ endothelial cells, ${ }^{43,44}$ and cardiomyocytes ${ }^{45,46}$ express oestrogen and androgen receptors. Indeed, single-cell transcriptomic analyses suggest that most cardiac cell types express sex hormone receptor genes, with individual cell types expressing specific receptors to varying degrees, and fibroblasts having the greatest level of transcripts corresponding to androgen and oestrogen receptors. ${ }^{24}$ Therefore, gonadal hormones may act on cardiac cells directly, but could also indirectly affect the heart through actions on noncardiac tissues including bone marrow ${ }^{47,48}$ and the spleen. ${ }^{49,50}$ These tissues can then affect the cardiac cellular environment by releasing secondary endocrine molecules or by direct recruitment of bone marrowor spleen-derived cells into the heart. ${ }^{51}$ Hormone replacement studies, as we have shown here, can address which specific hormones are critical in gonadectomy-induced remodelling of the cardiac cellular landscape. However, addressing the gaps in knowledge outlined above will necessitate the development of novel genetic models where hormone receptors can be modulated heart-specifically in a diverse range of cardiac cells. In addition to the action of hormones, sex chromosome effects on cardiac cellular heterogeneity are an alternative mechanism of cardiac cell regulation that remains to be explored. ${ }^{52}$ Nevertheless, the hormonal alteration experiments we have undertaken indicate that the cellular composition of the heart is plastic, and may be modulated to generate a salutary cellular signature as part of therapeutic applications.

The findings of this study should also be viewed in context of a number of other considerations. First, the extent to which sex-specific differences as described here, is identified in the humans and other species remains to be explored. Second, although we profile cellular heterogeneity using high-dimensional antibody panel validated by other studies, the precision of these markers for identifying specific cell populations has not been tested here. Third, our experiments were performed using a mouse diet that includes soybean- and alfalfa ingredients. These materials have detectable levels of phytoestrogens, ${ }^{53}$ and we have not measured phytoestrogens in the animal feed used in this study. The extent to which phytoestrogens modulate sex-specific cellular diversity of the heart remains to be explored, however, it should be taken into consideration that the presence of phytoestrogens may have influenced the results of this study. Finally, while female and male mice were housed separately, the oestrus cycles of female mice were not analysed. This may also affect the magnitude of sex-specific differences in the proportion of cardiac cell populations.

In summary, the findings made here identify distinct cellular signatures in female and male mouse hearts. These cellular differences may contribute to other sex-disparities of the heart such as in heart physiology, gene expression, and stress responses. Moreover, our results indicate that gonadal hormones are key drivers of sex differences in cardiac cellularity. Endocrine cues are not only potent regulators of the cardiac cellular landscape but, importantly, may also be manipulated in the future to therapeutically alter the cardiac cellular ecosystem.

\section{Supplementary material}

Supplementary material is available at Cardiovascular Research online. 


\section{Acknowledgements}

We acknowledge the use of JAX Flow Cytometry Core, Microscopy Core, Surgical Services, and Histology Service.

Conflict of interest: none declared.

\section{Funding}

This work is supported by National Health and Medical Research Council Ideas Grant (GNT1188503) to A.R.P. JAX Cores are supported by the Jackson Laboratory Cancer Center Core grant and the Leducq Foundation Transatlantic Network of Excellence in Cardiac Research (P30 CA034196) to N.A.R. The Australian Regenerative Medicine Institute is supported by grants from the State Government of Victoria and the Australian Government.

\section{Data availability}

Single-cell RNA-sequencing data analysed in this study are accessible from ArrayExpress (https://www.ebi.ac.uk/arrayexpress/) using the accession number E-MTAB-6173. All other data underlying this article are available in the article and in its Supplementary material online.

\section{References}

1. Blenck CL, Harvey PA, Reckelhoff JF, Leinwand LA. The importance of biological sex and estrogen in rodent models of cardiovascular health and disease. Circ Res 2016; 118:1294-1312.

2. Grandy SA, Howlett SE. Cardiac excitation-contraction coupling is altered in myocytes from aged male mice but not in cells from aged female mice Cardiac excitation-contraction coupling is altered in myocytes from aged male mice but not in cells from aged female mice. Am J Physiol Heart Circ Physiol 2006;291:2362-2370.

3. Isensee J, Witt H, Pregla R, Hetzer R, Regitz-Zagrosek V, Ruiz Noppinger P. Sexually dimorphic gene expression in the heart of mice and men. J Mol Med 2008;86:61-74.

4. Vijay V, Han T, Moland CL, Kwekel JC, Fuscoe JC, Desai VG. Sexual dimorphism in the expression of mitochondria-related genes in rat heart at different ages. PLoS One 2015; 10:e0117047

5. Skelly DA, Squiers GT, McLellan MA, Bolisetty MTMT, Robson P, Rosenthal NA, Pinto AR. Single-cell transcriptional profiling reveals cellular diversity and intercommunication in the mouse heart. Cell Rep Elsevier 2018;22:600-610.

6. Pinto AR, llinykh A, Ivey MJ, Kuwabara JT, D'antoni ML, Debuque RJ, Chandran A, Wang L, Arora K, Rosenthal NA, Tallquist MD. Revisiting cardiac cellular composition. Circ Res 2016;118:400-409.

7. Qiu P, Simonds EF, Bendall SC, Gibbs KD, Bruggner RV, Linderman MD, Sachs K, Nolan GP, Plevritis SK. Extracting a cellular hierarchy from high-dimensional cytometry data with SPADE. Nat Biotechnol 2011;29:886-891.

8. Butler A, Hoffman P, Smibert P, Papalexi E, Satija R. Integrating single-cell transcriptomic data across different conditions, technologies, and species. Nat Biotechnol 2018; 36:411-420.

9. Stuart T, Butler A, Hoffman P, Hafemeister C, Papalexi E, Mauck WM, Hao Y, Stoeckius M, Smibert P, Satija R. Comprehensive integration of single-cell data. Cell 2019;177:1888-1902.e21.

10. Bergmann O, Zdunek S, Alkass K, Druid H, Bernard S, Frisén J. Identification of cardiomyocyte nuclei and assessment of ploidy for the analysis of cell turnover. Exp Cell Res 2011;317:188-194.

11. Lerner DJ, Kannel WB. Patterns of coronary heart disease morbidity and mortality in the sexes: a 26-year follow-up of the Framingham population. Am Heart J 1986;111: 383-390.

12. Vaccarino V, Parsons L, Every NR, Barron HV, Krumholz HM. Sex-based differences in early mortality after myocardial infarction. N Engl J Med 1999;341:217-225.

13. Redfield MM, Jacobsen SJ, Borlaug BA, Rodeheffer RJ, Kass DA. Age- and genderrelated ventricular-vascular stiffening. Circulation 2005;112:2254-2262.

14. Guerra S, Leri A, Wang X, Finato N, Loreto C, Di Beltrami CA, Kajstura J, Anversa P. Myocyte death in the failing human heart is gender dependent. Circ Res 1999;85: 856-866.

15. Howlett SE. Age-associated changes in excitation-contraction coupling are more prominent in ventricular myocytes from male rats than in myocytes from female rats. Am J Physiol Heart Circ Physiol 2010;298:H659-H670.

16. Farrell SR, Ross JL, Howlett SE. Sex differences in mechanisms of cardiac excitationcontraction coupling in rat ventricular myocytes. Am J Physiol Heart Circ Physiol 2010; 299:H36-H45.
17. Huby RDJ, Glaves $P$, Jackson R. The incidence of sexually dimorphic gene expression varies greatly between tissues in the rat. PLoS One 2014;9:e115792.

18. Cavasin MA, Tao Z, Menon S, Yang X-P. Gender differences in cardiac function during early remodeling after acute myocardial infarction in mice. Life Sci 2004;75: 2181-2192.

19. Deegan DF, Karbalaei R, Madzo J, Kulathinal RJ, Engel N. The developmental origins of sex-biased expression in cardiac development. Biol Sex Differ 2019;10:46.

20. Gabel SA, Walker VR, London RE, Steenbergen C, Korach KS, Murphy E. Estrogen receptor beta mediates gender differences in ischemia/reperfusion injury. J Mol Cell Cardiol 2005;38:289-297.

21. Wang M, Baker L, Tsai BM, Meldrum KK, Meldrum DR. Sex differences in the myocardial inflammatory response to ischemia-reperfusion injury. Am J Physiol Endocrinol Metab 2005;288:E321-E326.

22. Fliegner D, Schubert C, Penkalla A, Witt H, Kararigas G, Dworatzek E, Staub E, Martus P, Noppinger PR, Kintscher U, Gustafsson J-Å, Regitz-Zagrosek V, Fliegner D, Schubert C, Penkalla A, Witt H, Kararigas $G$. Female sex and estrogen receptor- $\beta$ attenuate cardiac remodeling and apoptosis in pressure overload. Am J Physiol Regul Integr Comp Physiol 2010;298:1597-1606.

23. Fang L, Gao X-M, Moore X-L, Kiriazis H, Su Y, Ming Z, Lim YL, Dart AM, Du X-J. Differences in inflammation, MMP activation and collagen damage account for gender difference in murine cardiac rupture following myocardial infarction. J Mol Cell Cardiol 2007;43:535-544.

24. McLellan MA, Skelly DA, Dona MSI, Squiers GT, Farrugia GE, Gaynor TL, Cohen CD, Pandey R, Diep H, Vinh A, Rosenthal NA, Pinto AR. High-resolution transcriptomic profiling of the heart during chronic stress reveals cellular drivers of cardiac fibrosis and hypertrophy. Circulation 2020; circulationaha.119.045115.

25. Forte E, Skelly DA, Chen M, Daigle S, Morelli KA, Hon O, Philip VM, Costa MW, Rosenthal NA, Furtado MB. Dynamic interstitial cell response during myocardial infarction predicts resilience to rupture in genetically diverse mice. Cell Rep 2020;30: 3149-3163.e6.

26. Fan D, Takawale A, Lee J, Kassiri Z. Cardiac fibroblasts, fibrosis and extracellular matrix remodeling in heart disease. Fibrogenesis Tissue Repair 2012;5:15-13.

27. Gourdie RG, Dimmeler S, Kohl P. Novel therapeutic strategies targeting fibroblasts and fibrosis in heart disease. Nat Rev Drug Discov 2016;15:620-638.

28. Furtado MB, Costa MW, Pranoto EA, Salimova E, Pinto AR, Lam NT, Park A, Snider P, Chandran A, Harvey RP, Boyd R, Conway SJ, Pearson J, Kaye DM, Rosenthal NA. Cardiogenic genes expressed in cardiac fibroblasts contribute to heart development and repair. Circ Res 2014;114:1422-1434.

29. Cartledge JE, Kane C, Dias P, Tesfom M, Clarke L, Mckee B, Ayoubi S, Al Chester A Yacoub MH, Camelliti P, Terracciano CM. Functional crosstalk between cardiac fibroblasts and adult cardiomyocytes by solublemediators. Cardiovasc Res 2015;105: 260-270.

30. Kay E, Gomez-Garcia L, Woodfin A, Scotland RS, Whiteford JR. Sexual dimorphisms in leukocyte trafficking in a mouse peritonitis model. J Leukoc Biol 2015;98:805-817.

31. Hofmann U, Frantz S. Role of lymphocytes in myocardial injury, healing, and remodeling after myocardial infarction. Circ Res 2015;116:354-367.

32. Weirather J, Hofmann UDW, Beyersdorf N, Ramos GC, Vogel B, Frey A, Ertl G, Kerkau T, Frantz S. Foxp3+ CD4+ T cells improve healing after myocardial infarction by modulating monocyte/macrophage differentiation. Circ Res 2014;115:55-67.

33. Tang T-T, Yuan J, Zhu Z-F, Zhang W-C, Xiao H, Xia N, Yan X-X, Nie S-F, Liu J, Zhou S-F, Li J-J, Yao R, Liao M-Y, Tu X, Liao Y-H, Cheng X. Regulatory T cells ameliorate cardiac remodeling after myocardial infarction. Basic Res Cardiol 2012;107:232.

34. Yang Z, Day Y-J, Toufektsian M-C, Xu Y, Ramos SI, Marshall MA, French BA, Linden J. Myocardial infarct-sparing effect of adenosine $A 2 A$ receptor activation is due to its action on CD4+ T lymphocytes. Circulation 2006;114:2056-2064.

35. Timmers L, Pasterkamp G, Hoog VC, de Arslan F, Appelman Y, de Kleijn DPV. The innate immune response in reperfused myocardium. Cardiovasc Res 2012;94:276-283.

36. Huang GN, Thatcher JE, McAnally J, Kong Y, Qi X, Tan W, DiMaio JM, Amatruda JF, Gerard RD, Hill JA, Bassel-Duby R, Olson EN. C/EBP transcription factors mediate epicardial activation during heart development and injury. Science 2012;338: 1599-1603.

37. Duilio C, Ambrosio G, Kuppusamy P, DiPaula A, Becker LC, Zweier JL. Neutrophils are primary source of $\mathrm{O} 2$ radicals during reperfusion after prolonged myocardial ischemia. Am J Physiol Heart Circ Physiol 2001;280:H2649-H2657.

38. Lavine KJ, Pinto AR, Epelman S, Kopecky BJ, Clemente-Casares X, Godwin J, Rosenthal N, Kovacic JC. The macrophage in cardiac homeostasis and disease. J Am Coll Cardiol 2018;72:2213-2230.

39. Campbell L, Emmerson E, Williams H, Saville CR, Krust A, Chambon P, Mace KA Hardman MJ. Estrogen receptor-alpha promotes alternative macrophage activation during cutaneous repair. J Invest Dermatol 2014;134:2447-2457.

40. Lai JJ, Lai KP, Chuang KH, Chang P, Yu IC, Lin WJ, Chang C. Monocyte/macrophage androgen receptor suppresses cutaneous wound healing in mice by enhancing local TNF- $\alpha$ expression. J Clin Invest 2009;119:3739-3751.

41. Lee H-W, Eghbali-Webb M. Estrogen enhances proliferative capacity of cardiac fibroblasts by estrogen receptor- and mitogen-activated protein kinase-dependent pathways. J Mol Cell Cardiol 1998;1368:1359-1368.

42. Stewart JA, Cashatt DO, Borck AC, Brown JE, Carver WE. 17beta-estradiol modulation of angiotensin II-stimulated response in cardiac fibroblasts. J Mol Cell Cardiol 2006;41:97-107. 
43. Chakrabarti S, Morton JS, Davidge ST. Mechanisms of estrogen effects on the endothelium: an overview. Can J Cardiol 2014;30:705-712.

44. Cai J, Hong Y, Weng C, Tan C, Imperato-Mcginley J, Zhu Y-S. Androgen stimulates endothelial cell proliferation via an androgen receptor/VEGF/cyclin A-mediated mechanism. Am J Physiol Heart Circ Physiol 2011;300: H1210-H1221.

45. Luo T, Kim JK. The role of estrogen and estrogen receptors on cardiomyocytes: an overview. Can J Cardiol 2016:32:1017-1025.

46. Cavasin MA, Sankey SS, Yu AL, Menon S, Yang XP. Estrogen and testosterone have opposing effects on chronic cardiac remodeling and function in mice with myocardial infarction. Am J Physiol Heart Circ Physiol 2003;284:H1560-H1569.

47. Medina KL, Garrett KP, Thompson LF, Rossi MI, Payne KJ, Kincade PW. Identification of very early lymphoid precursors in bone marrow and their regulation by estrogen. Nat Immunol 2001;2:718-724.

48. Hamada H, Kim MK, Iwakura A, li M, Thorne T, Qin G, Asai J, Tsutsumi Y, Sekiguchi H, Silver M, Wecker A, Bord E, Zhu Y, Kishore R, Losordo DW. Estrogen receptors alpha and beta mediate contribution of bone marrow-derived endothelial progenitor cells to functional recovery after myocardial infarction. Circulation 2006;114: 2261-2270.

49. Erlandsson MC, Ohlsson C, Gustafsson JA, Carlsten H. Role of oestrogen receptors alpha and beta in immune organ development and in oestrogen-mediated effects on thymus. Immunology 2001;103:17-25.

50. Zhang J, Pugh TD, Stebler B, Ershler WB, Keller ET. Orchiectomy increases bone marrow interleukin-6 levels in mice. Calcif Tissue Int 1998;62:219-226.

51. Molawi K, Wolf Y, Kandalla PK, Favret J, Hagemeyer N, Frenzel K, Pinto AR, Klapproth K, Henri S, Malissen B, Rodewald H-R, Rosenthal NA, Bajenoff M, Prinz M, Jung S, Sieweke $\mathrm{MH}$. Progressive replacement of embryo-derived cardiac macrophages with age. J Exp Med 2014;211:2151-2158.

52. Arnold AP. The end of gonad-centric sex determination in mammals. Trends Genet 2012;28:55-61.

53. Thigpen JE, Setchell KDR, Kissling GE, Locklear J, Caviness GF, Whiteside T, Belcher SM, Brown NM, Collins BJ, Lih FB, Tomer KB, Padilla-Banks E, Camacho L, Adsit FG, Grant M. The estrogenic content of rodent diets, bedding, cages, and water bottles and its effect on bisphenol a studies. J Am Assoc Lab Anim Sci 2013;52:130-141.

\section{Translational perspective}

The significance of endocrine cues, particularly oestrogens, on cardiac physiology and disease pathogenesis has been known for decades. This work highlights basal sex differences in cardiac cellularity and shows that endocrine signalling is necessary to maintain the cellular diversity of the heart. By studying the impact of cardiac cellular diversity on heart physiology and stress responses, and its tractability by factors such as hormones, the cardiac cellular landscape may be modulated for targeted therapeutic approaches in the future.

\section{Corrigendum}

doi:10.1093/cvr/cvab233

Online publish-ahead-of-print 27 July 2021

\section{Corrigendum to: Progress in aorta and peripheral cardiovascular disease research}

Lucia Mazzolai, Adriano Alatri, Alessandra Bura Rivière, Marco De Carlo, Christian Heiss, Christine Espinola-Klein, Oliver Schlager, Henrik Sillesen, Daniel Staub, José F Rodriguez-Palomares, Aline Verstraeten, Victor Aboyans on behalf of the WG on aorta and peripheral vascular diseases

\section{Cardiovascular Research, cvab144, https://doi.org/10.1093/cvr/cvab144}

When this paper first published, one author erroneously appeared as José R Palomares. This author should have been José $F$ RodriguezPalomares. This has now been corrected online. 